\title{
A conserved role of Shp2 in regulation of human and mouse ES cell differentiation
}

\author{
Dongmei $\mathrm{Wu}^{1}$, Yuhong Pang ${ }^{1}$, Yuehai $\mathrm{Ke}^{1}$, Zhao He ${ }^{1}$, Gen-Sheng Feng ${ }^{1}$ \\ ${ }^{1}$ Institute for Biomedical Research, Xiamen University, Xiamen, China; Burnham Institute for Medical Research, La Jolla, CA \\ 92037, USA
}

The establishment of human embryonic stem cell (hESC) lines has generated a great deal of excitement in the fields of stem cell biology and regenerative medicine. It has been hoped that hESCs and somatic stem cells could be potential sources of cells to regenerate or rejuvenate damaged tissues. Yet studies on the biology of hESCs have been hindered by difficulties in manipulating this type of cells in culture in vitro. This is largely due to lack of full understanding of the molecular and cellular basis for maintenance of hESC pluripotency. Since mESCs have received extensive attentions in the past two decades and relatively more information is available, characterization of hESCs has been naturally conducted by referencing mESC properties. Recent studies from a number of laboratories have suggested distinct biological properties and molecular signaling mechanisms between human and mouse embryonic stem cells (hESCs and mESCs). Here we report that Shp2, an intracellular tyrosine phosphatase with two SH2 domains, has a conserved role in promoting signals resulting in differentiation of both hESCs and mESCs. Homozygous ablation of Shp2 in mESCs resulted in impaired differentiation into all three germ layer cell lineages accompanied by improved self-renewal. siRNA-mediated Shp2 knockdown in hESCs resulted in a very similar phenotype of impaired differentiation. Furthermore, small molecule inhibitors for Shp2 enzyme suppressed both hESC and mESC differentiation in vitro. Shp2 modulates LIF and BMP signals flowing through Erk, Stat3 and Smad pathways in mESCs and also regulates FGF and BMP signaling through the Erk and Smad pathways in hESCs. These results reveal a common signaling mechanism shared by hESCs and mESCs via Shp2 modulation of overlapping and distinct pathways. Pharmaceutical intervention of Shp2 activity might be a practical means for amplification of hESCs in vitro.

Keywords: Shp2 tyrosine phosphatase, signaling mechanism, embryonic stem cells, pluripotency

Cell Research (2008) 18:s27. doi: 10.1038/cr.2008.117; published online 4 August 2008

Correspondence: Gen-Sheng Feng

E-mail: gfeng@burnham.org

Gen-Sheng Feng is a professor at the Burnham Institute for Medical Research, and the University of California San Diego, La Jolla, California. Dr Feng is also a professor at the Institute for Biomedical Research, Xiamen University. He earned his $\mathrm{PhD}$ in Indiana University Bloomington in 1990, and received postdoctoral training in the Hospital for Sick Children and Mount Sinai Hospital, Toronto, Canada. In 1994 he was appointed as an assistant professor at Indiana University School of Medicine, and was promoted to associate professor in 1999. Dr Feng moved his lab to the Burnham Institute in 2000. Research in Dr Feng's lab is focused on dissection of molecular signaling mechanisms controlling pluripotency of human and mouse embryonic stem cells, and adult stem cells. His group is also interested in deciphering the molecular basis for metabolic diseases. 